\title{
External validation of the eighth edition of the TNM classification for lung cancer in 3,611 surgically treated patients at a single institution
}

\author{
Jia Wang, Nan Wu, Chao Lv, Yue Yang \\ Department of Thoracic Surgery II, Peking University Cancer Hospital \& Institute, Beijing 100142, China \\ Contributions: (I) Conception and design: Y Yang, N Wu; (II) Administrative support: N Wu; (III) Provision of study materials or patients: Y Yang; \\ (IV) Collection and assembly of data: J Wang, C Lv; (V) Data analysis and interpretation: J Wang; (VI) Manuscript writing: All authors; (VII) Final \\ approval of manuscript: All authors. \\ Correspondence to: Yue Yang. Key Laboratory of Carcinogenesis and Translational Research (Ministry of Education/Beijing), Department of \\ Thoracic Surgery II, Peking University Cancer Hospital \& Institute, No. 52, Fucheng Avenue, Haidian District, Beijing 100142, China. \\ Email: surgeonwangjia@163.com.
}

\begin{abstract}
Background: A new revision of the tumor, node, metastasis (TNM) classification for lung cancer has been proposed by the International Association for the Study of Lung Cancer (IASLC), but external validation for it is required. This study aimed to evaluate stage groupings in the 8th edition of the TNM classification in an independent Chinese cohort.

Methods: We retrospectively analyzed 3,611 patients who were diagnosed as stage I to IV non-small cell lung cancer (NSCLC) and who received surgical treatment at our institute in China between October 2009 and August 2017. Long-rank tests were used to compare survival between two adjacent stage groups.

Results: Based on the 8th edition of the TNM classification, differences between every 2 adjacent stage groups were found to be significant except between Ia1 and Ia2 (P=0.062), and between IIIc and IVa $(\mathrm{P}=0.063)$. Significant differences were found between every 2 adjacent categories stratified by the $\mathrm{T}$ and $\mathrm{N}$ descriptors. Additionally, significant differences were found between $\mathrm{M} 0$ and $\mathrm{M} 1 \mathrm{a}(\mathrm{P}<0.001)$, while no significant difference was observed between M1a and M1b ( $\mathrm{P}=0.092)$.
\end{abstract}

Conclusions: Our study provides an external validation of the stage groupings in the 8th edition of the TNM staging system in surgically treated Chinese patients with NSCLC.

Keywords: Validation study; neoplasm staging; tumor, node, metastasis classification (TNM classification); nonsmall cell lung cancer (NSCLC); Chinese patients

Submitted Dec 30, 2019. Accepted for publication Jan 14, 2020.

doi: $10.21037 /$ atm.2020.01.128

View this article at: http://dx.doi.org/10.21037/atm.2020.01.128

\section{Introduction}

Lung cancer is the leading cause of cancer death and a major public health problem worldwide (1). Approximately 85\% of lung cancer cases are non-small cell lung cancer (NSCLC) with a 5 -year overall survival (OS) rate of $15 \%$ (2). The tumor, node, metastasis (TNM) staging system has been commonly used in NSCLC to predict prognosis and make treatment decisions (3). Thus, accurate staging of the disease is essential in its management since this staging is responsible for identifying stage-specific treatment strategies and providing an estimation of the patient's prognosis (4).

The 7th edition of the TNM staging system for lung cancer was released in 2009 (5), and in January 2017, the 8th edition was published by the American Joint Committee on Cancer (AJCC) and the Union for International Cancer Control (UICC) (6). This edition was based on the new International Association for the Study of Lung Cancer 
Page 2 of 8

Table 1 Demographics and characteristics of the patients $(\mathrm{N}=3,611)$

\begin{tabular}{ll}
\hline Characteristics & Value, N (\%) \\
\hline Gender & \\
Male & $2,094(58.0)$ \\
Female & $1,517(42.0)$ \\
Median age, y [range] & $62 \pm 7.2[24-89]$ \\
Surgical approach & \\
Lobectomy & $2,175(60.2)$ \\
Wedge resection & $813(22.5)$ \\
Segmentectomy & $338(9.4)$ \\
Bilobectomy & $112(3.1)$ \\
Pneumonectomy & $108(3.0)$ \\
Exploratory thoracotomy & $65(1.8)$ \\
Perioperative therapy & \\
Neoadjuvant chemotherapy & $328(9.1)$ \\
Neoadjuvant targeted therapy & $286(7.9)$ \\
Adjuvant chemotherapy & $986(27.3)$ \\
Adjuvant targeted therapy & $351(9.7)$ \\
Postoperative radiotherapy & $483(13.4)$ \\
Histologic diagnosis & $2,796(77.4)$ \\
Adenocarcinoma & $602(16.7)$ \\
Squamous carcinoma & $81(2.2)$ \\
\hline
\end{tabular}

(IASLC) database comprising information collected from 94,708 patients diagnosed as lung cancer from 1999 to 2010 (7). Though a large number of patients from Asia were included, mainly from Japan, less than $1 \%$ of patients were from China (8). This proportion is small given that China has the highest incidence of lung cancer worldwide and the clinical characteristics of Chinses patients are different from those of Japanese patients (9). Moreover, in the past decade, targeted therapy and immunotherapy have played an increasingly essential part in changing the prognosis of patients with lung cancer and clinical treatment modes (10). Therefore, an external validation of the 8th edition TNM staging system among Chinese patients is urgently required.

Our study evaluated the effects of the reclassification

\section{Wang et al. Evaluation of the TNM classification for lung cancer}

from the 7 th to the 8 th edition on stage groupings and survival characteristics of 3611 patients who received pneumonectomy at a single institution in a given period to evaluate the clinical significance of the new staging system and to discuss potential problems in Chinese patients.

\section{Methods}

\section{Study design and patients}

Our study was approved by the Institutional Review Board at the Peking University Cancer Hospital. Patient consent was not required since no human subjects participated in our study. We collected the medical records of 3,611 patients who were diagnosed as stage I to IV NSCLC and who underwent tumor resection or lymph node dissection or sampling at the Department of Thoracic Surgery II, Peking University Cancer Hospital, between October 2009 and August 2017.

\section{Follow-up}

Abdominal and supraclavicular ultrasound scanning, bone scintigraphy, computed tomography (CT), or brain magnetic resonance imaging (MRI) were performed routinely before surgery to assess the extent of the disease. CT scans/ positron emission tomography were not used routinely due to the expense. Patients were postoperatively examined at 3 -month intervals for the first 2 years, every 6 months for another 3 years and yearly thereafter to check for survival and recurrence in a median follow-up period of 62 months (range, 1-128 months).

\section{Statistical analysis}

Survival curves were calculated by Kaplan-Meier method. The significance of survival was determined using log-rank tests. $\mathrm{P}<0.05$ was considered statistically significant. SPSS software version 20.0 (IBM, Chicago, IL, USA) was used for all analyses.

\section{Results}

\section{Patient demographics and characteristics}

Patient demographics and characteristics are listed in Table 1. A total of 3,611 subjects (2,094 males and 1,517 females) with a median age of $62 \pm 7.2$ years (range, $24-89$ ) years were enrolled in our study. 
A

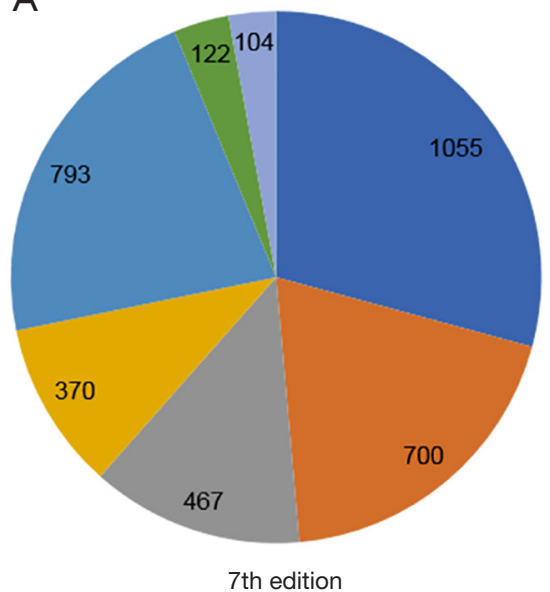

B

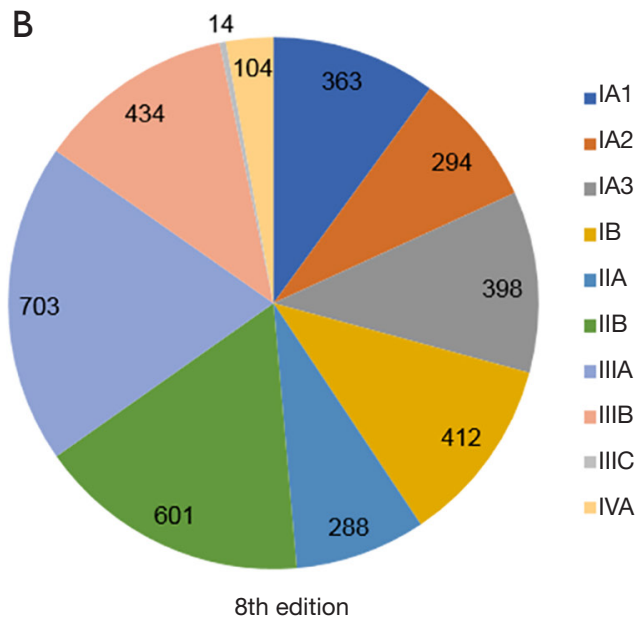

Figure 1 Comparison of distribution in stage subgroups in the 7th (A) and the 8th (B) editions of the TNM classification. TNM, tumor, node, metastasis.

Among all the patients, lobectomy was the main surgical procedure $(60.2 \%)$. The remaining patients underwent the following surgical treatments: wedge resection in 813 patients (22.5\%), segmentectomy in 338 cases $(9.4 \%)$, bilobectomy in 112 cases (3.1\%), pneumonectomy in 108 cases $(3.0 \%)$, and exploratory thoracotomy in 65 cases (1.8\%). Meanwhile, neoadjuvant therapy was performed in $614(17 \%)$ patients, among whom $328(9.1 \%)$ and 286 (7.9\%) received neoadjuvant chemotherapy or neoadjuvant targeted therapy, respectively, with $986(27.3 \%)$ and 351 $(9.7 \%)$ patients at stage IB or higher stages undergoing adjuvant chemotherapy or adjuvant targeted therapy, respectively. In addition, postoperative radiotherapy was performed in $483(13.4 \%)$ patients.

In total, 2,796 patients were diagnosed with adenocarcinoma, 602 with squamous cell carcinoma, 81 with adenosquamous carcinoma, 73 with large cell carcinoma, and 59 with other types of lung cancer. Cardiac evaluation and pulmonary function testing were performed before surgery.

\section{Distribution of pathologic stages}

Based on the 7th edition, a total of 3,611 subjects were categorized into the following categories: 1,055 (29.2\%) at pStage IA, $700(19.4 \%)$ at pStage IB, $467(12.9 \%)$ at pStage IIA, $370(10.2 \%)$ at pStage IIB, 793 (22.0\%) at pStage IIIA, $122(3.4 \%)$ at pStage IIIB, and $104(2.9 \%)$ at pStage IV (Figure 1A). Under the 8 th edition, all patients included in this study were divided into the following categories: pStage
IA1 $(\mathrm{n}=363,10.1 \%)$, pStage IA2 $(\mathrm{n}=294,8.1 \%)$, pStage IA3 ( $\mathrm{n}=398,11.0 \%)$, pStage IB ( $\mathrm{n}=412,11.4 \%)$, pStage IIA $(\mathrm{n}=288,8.0 \%)$, pStage IIB $(\mathrm{n}=601,16.6 \%)$, pStage IIIA $(\mathrm{n}=703,19.5 \%)$, pStage IIIB $(\mathrm{n}=434,12.0 \%)$, pStage IIIC $(\mathrm{n}=14,0.4 \%)$, and pStage IVA $(\mathrm{n}=104,2.9 \%)$ (Figure 1B). Overall, 1,331 (36.9\%) patients were redefined as a new pStage and shifted to a higher pStage in the 8th edition; these shifts of stage groupings are shown in Table 2.

\section{Survival analysis}

Figure 2 depicts the survival curves and the 5 -year survival rates according to the 7 th and the 8 th editions of the TNM classification. The survival curves show a stepwise deterioration as the pStage progresses except in pStage IIIb and pStage IV and significant differences between every 2 adjacent stage groups can be seen in the 7 th edition (Figure 2A). Under the 8 th edition, the same results can observed as the pStage progresses except in pStage IIIC and $\mathrm{IVa}$, while differences between every 2 adjacent stage groups except between Ia 1 and Ia2 ( $\mathrm{P}=0.062)$, and between IIIc and $\mathrm{IVa}(\mathrm{P}=0.063)$ are significant (Figure $2 B)$.

The survival curves and the 5 -year survival rates stratified by $T$ classification in accordance with the 7 th and the 8 th editions are presented in Figure 3. Both the survival curves and the 5-year survival rates display stepwise deterioration as the $T$ classification progresses, and significant differences can be observed between every 2 adjacent categories.

For the $\mathrm{N}$ classification summarized in Figure 4, the survival curves and the 5 -year survival rates based on the 
Table 2 Stage shift of the patients when applying the 8th edition of the TNM classification

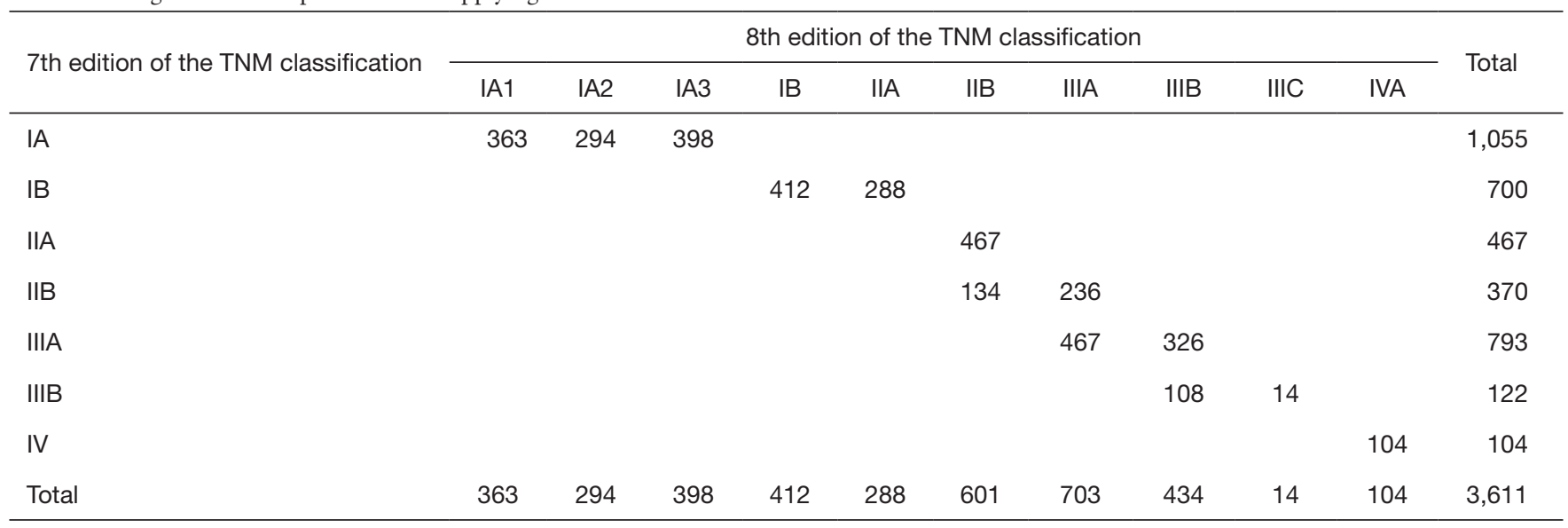
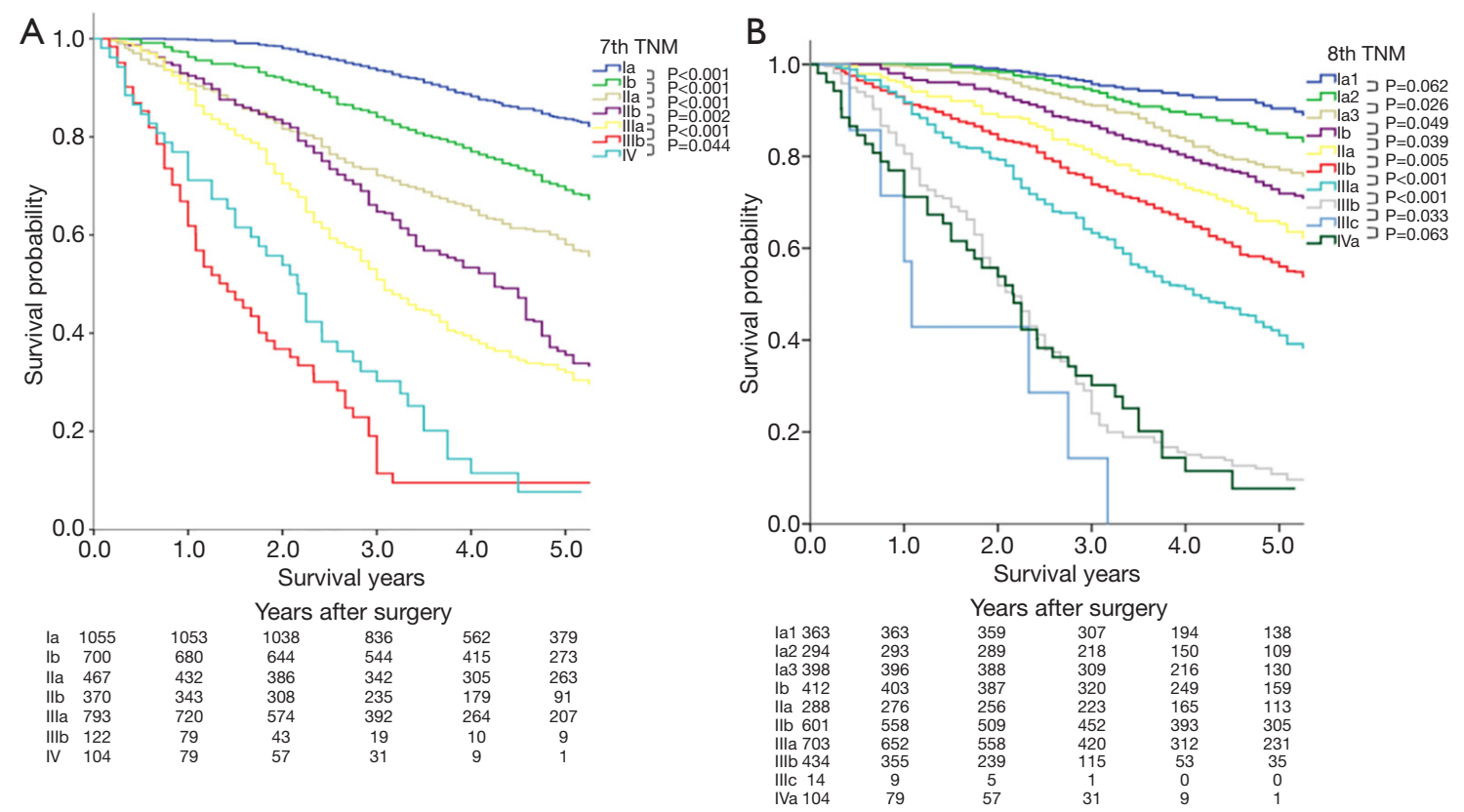

Figure 2 Survival curves of the patients based on the 7th (A) and 8th (B) editions of the TNM staging system for lung cancer. The P values and the 5-year survival rate between every 2 adjacent subgroups are indicated. TNM, tumor, node, metastasis.

8th edition decrease as the $\mathrm{N}$ classification progresses, and a significant difference can be seen between every 2 adjacent categories.

For the $M$ classification, the survival curves and the 5-year survival rates based on the 7 th and the 8 th editions are depicted in Figure 5. Significant differences between every two adjacent groups can be identified in the 7 th edition $(\mathrm{P}<0.001)$ (Figure $5 A)$. For the 8 th edition, significant difference between $\mathrm{M} 0$ and $\mathrm{M} 1 \mathrm{a}(\mathrm{P}<0.001)$ are evident; however, no significant difference can be observed between
M1a and M1b $(\mathrm{P}=0.092)$ (Figure 5B). Since all patients included in this study were surgical cases, no data for M1c patients were available.

\section{Discussion}

With the deepening understanding of lung cancer and the progress of modern medicine, the staging system has undergone significant revisions which could lead to changes in therapeutic strategy (11). With the 7 th 


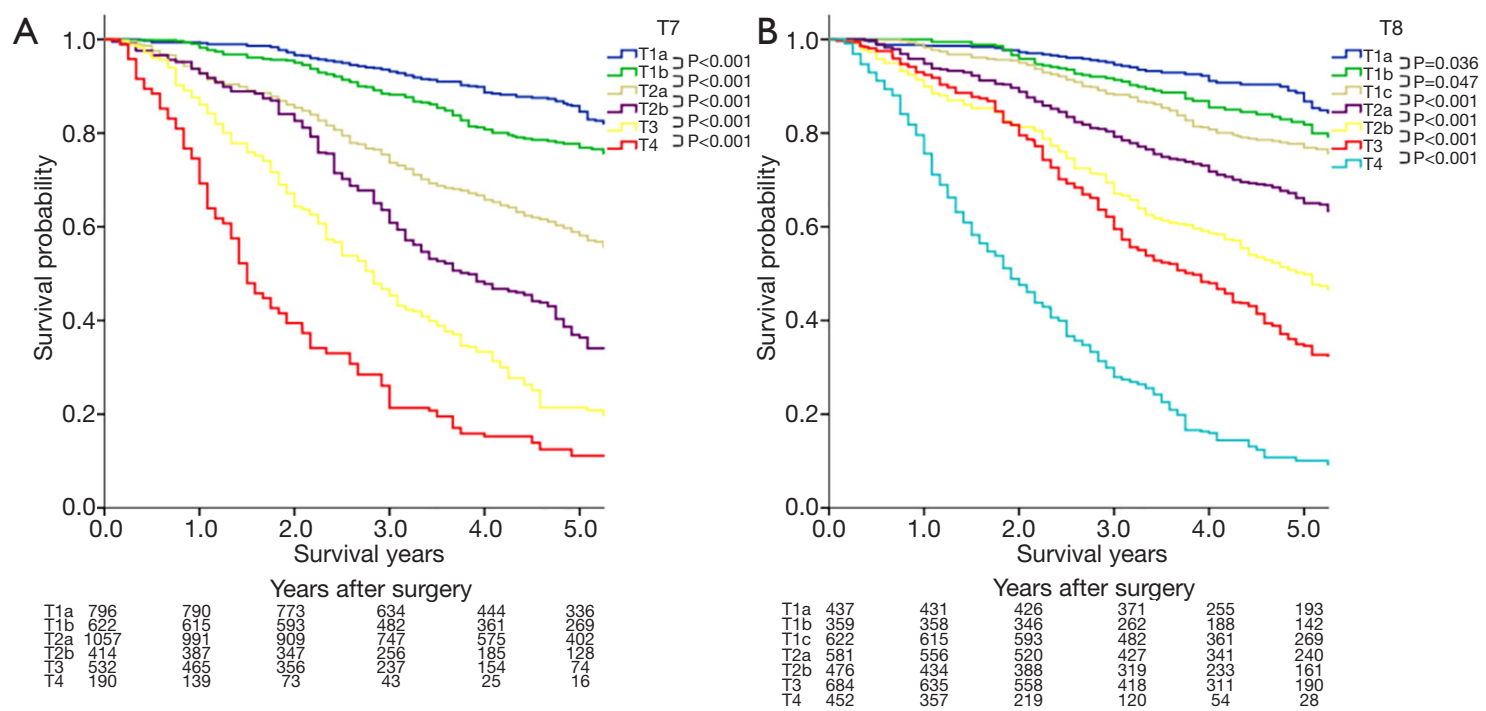

Figure 3 Survival curves of the patients stratified by $\mathrm{T}$ descriptors according to the 7 th $(\mathrm{A})$ and 8 th $(\mathrm{B})$ editions. The $\mathrm{P}$ values and the 5 -year survival rate between every 2 adjacent subgroups are indicated.

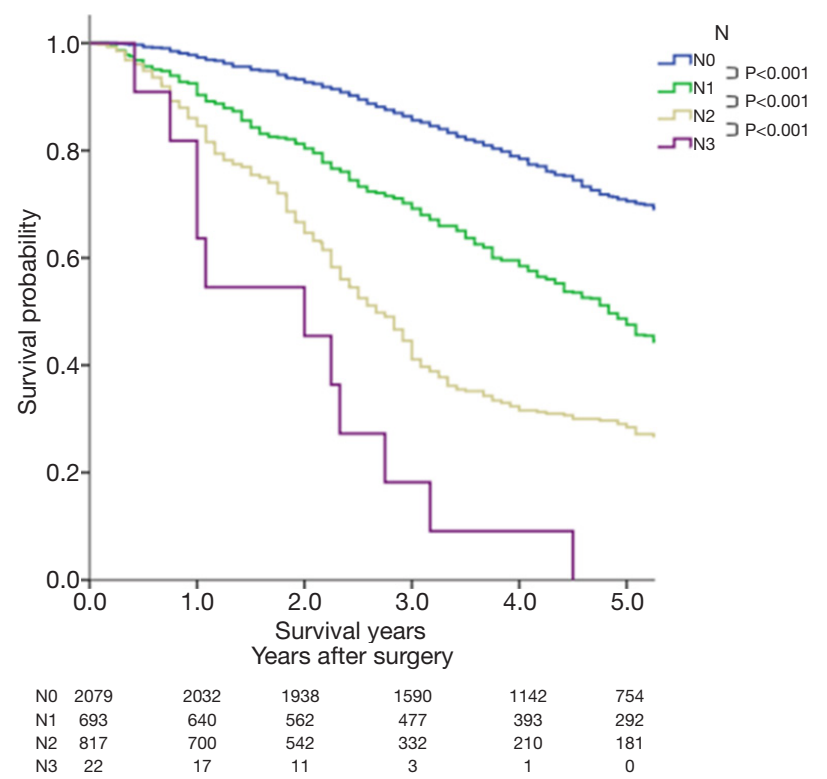

Figure 4 Survival curves of the patients based on $\mathrm{N}$ descriptors in the 8 th edition. The 5 -year survival rate and the $\mathrm{P}$ values between every 2 adjacent subgroups are indicated.

edition of the TNM staging system, the controversy has centered around whether patients at stage IB need adjuvant chemotherapy (12). Since the 8 th edition of the TNM staging system defined the tumor size as $>3$ and $\leq 4 \mathrm{~cm}$ for T2a and $>4 \mathrm{~cm}$ for stage IIA (13), stage IB patients in the 7 th edition were divided into stage IB and stage IIA in the 8th edition. Research has shown that for stage IB NSCLC patients classified in the 7 th edition, adjuvant chemotherapy is superior to observation alone (14). By contrast, under the 8th edition, adjuvant chemotherapy had worse OS for stage IB NSCLC patients, but the opposite results were found in stage IIA NSCLC patients (15). The same conclusion was reached in the CALBG 9633 trial which has been the only randomized trial of adjuvant chemotherapy for lung cancer patients to focus exclusively on those at stage IB according to the 7 th edition $(16,17)$. The CALBG 9633 study failed to show OS benefits for adjuvant chemotherapy among stage IB patients ( $\mathrm{HR}=0.83, \mathrm{P}=0.12$ ), whereas subset analysis revealed that chemotherapy improved OS for those with tumors $\geq 4 \mathrm{~cm}$ ( $\mathrm{HR}=0.66, \mathrm{P}=0.04)$ (17). Thus, the 8 th edition is more accurate than the 7 th edition in defining whether stage IB patients should take adjuvant chemotherapy.

It also remains controversial whether lobectomy or segmentectomy is the best surgical method for ground-glass opacity (18). Lung Cancer Study Group (LCSG) 082 was designed to compare the survival outcome of lobectomy with that of sublobectomy by using the $3 \mathrm{~cm}$ diameter of tumors (T1N0 in the 6th edition) as the selection criterion (19). Although no significant difference in OS between the 2 groups was found, the limited resection group had a significantly higher 3-year local recurrence rate than the lobectomy group, suggesting that it was unreasonable to use $3 \mathrm{~cm}$ diameter of the tumor as the criterion (19). In 

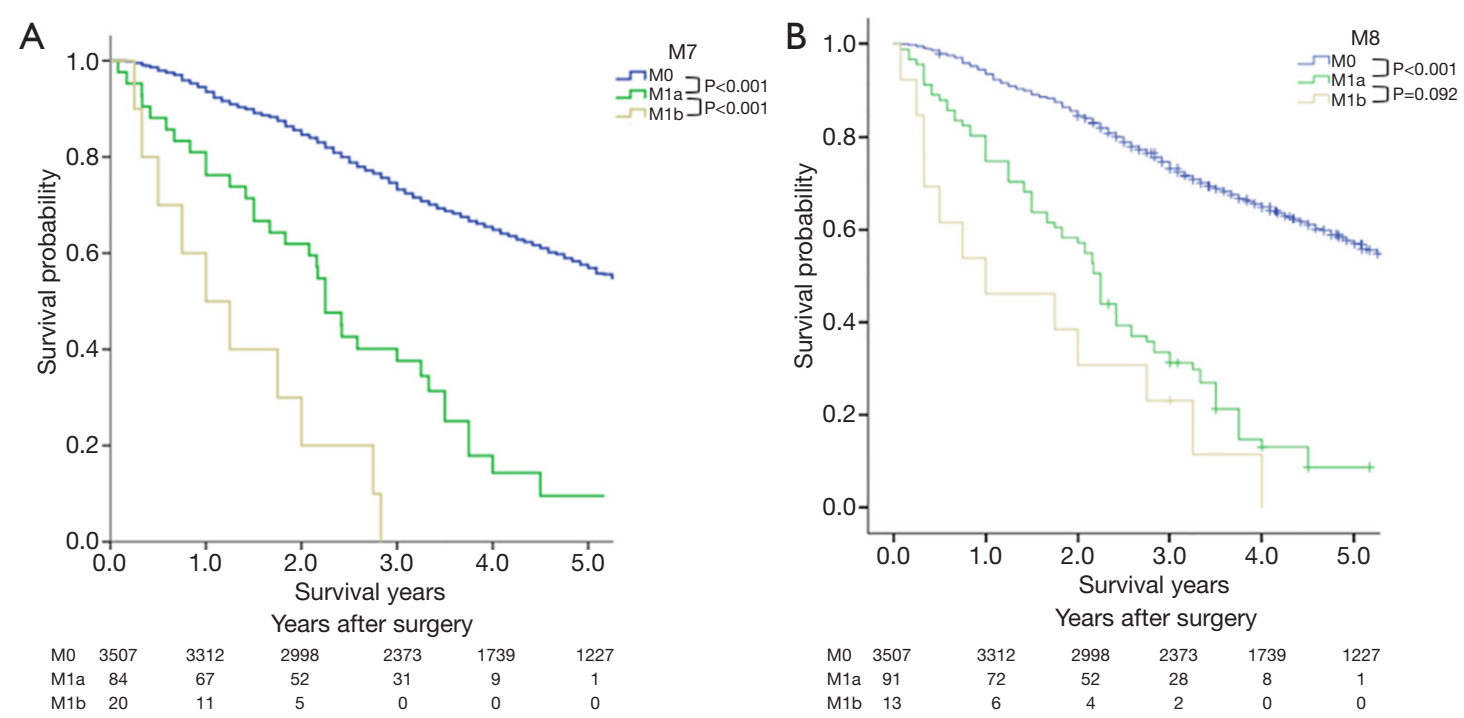

Figure 5 Survival curves based on $M$ classification in line with the 7 th $(\mathrm{A})$ and 8 th $(\mathrm{B})$ editions. The $\mathrm{P}$ values and the 5 -year survival rate between every 2 adjacent subgroups are indicated.

accordance with the 7th edition of TNM staging system, 3 studies of sublobectomy including CALGB140503, JCOG0802, and EORTC1223 have been carried out (20). These studies used $2 \mathrm{~cm}$ as the criterion for group selection (T1N0 in the 7th edition), but are still not concluded (20). The 8 th edition highlighted the role of tumor size in the prognosis of patients (21). With $1 \mathrm{~cm}$ as the dividing point, the IA period of the 7th edition was divided into IA1, IA2, and IA 3 in the 8 th edition (21). Similarly, the stage IA in the 7 th edition was divided into IA1, IA2, and IA 3 in accordance with the 8 th edition. Although no significant difference between IA1 and IA2 $(\mathrm{P}=0.062)$ was found, the survival curves of IA1, IA2, and IA3 showed stepwise deterioration as the pStage progressed, providing more supporting data for the subdivision of stage I lung cancer in the 8 th edition. Under the guidance of the 8 th edition, the discussion with respect to lobectomy or sublobectomy for ground-glass opacity may enter a new era.

T3N2M0 moved from stage IIIA in the 7 th edition to IIIB in the 8th due to the worse outcome (22). This study revealed that although the OS of T3N2M0 patients was obviously worse than that of T1N2M0 IIIA patients $(\mathrm{P}<0.001)$, the survival curve of $\mathrm{T} 3 \mathrm{~N} 2 \mathrm{M} 0$ patients remained close to that of T2N2M0 IIIA patients, and T3N2M0 patients displayed significantly better OSs compared with T4N2M0 IIIB patients $(\mathrm{P}=0.008)$. Clearly, these results are not supportive of the revision on T3N2M0 in the 8 th edition.
It has been shown that there is a correlation between prognosis and tumor size (23). Among the 8th edition stages, $\mathrm{T}$ stage had the greatest change; most notably, $\mathrm{T}$ $\leq 3 \mathrm{~cm}$ was further refined in the following fashion: T1a was defined as maximum tumor diameter $\leq 1 \mathrm{~cm}, \mathrm{~T} 1 \mathrm{~b}$ as $1 \mathrm{~cm}<$ maximum tumor diameter $\leq 2 \mathrm{~cm}$, and T1c as $2 \mathrm{~cm}$ $<$ maximum tumor diameter $\leq 3 \mathrm{~cm}$ (24). In this study, we observed significant differences in prognosis between $\mathrm{T} 1 \mathrm{a}$ and T1b $(\mathrm{P}=0.036)$ and between T1b and T1c $(\mathrm{P}=0.047)$, providing more evidence supportive of the further subdivision of $\mathrm{T}<3 \mathrm{~cm}$ in the 8 th edition.

The accepted $\mathrm{N}$ descriptors of the 7 th edition continue to be used in the 8th edition (25). Remarkably, the high prognostic value of the $\mathrm{N}$ subclassification was confirmed in our study.

In the 8 th edition, the M1 category was further divided into intrathoracic metastasis (M1a), single extrathoracic metastasis (M1b), and multiple extrathoracic metastases (M1c) (26). M1a includes pericardial or pleural dissemination and separate tumor nodules in the contralateral lobe (27). Since all our patients were surgical cases, no patients at M1c were included in the study. While 91 patients at M1a underwent only thoracic exploration without removing the primary tumor due to pleural dissemination revealed by intraoperative exploration, in 13 patients, M1b oligometastases (such as single brain or single adrenal metastases) were found before the resection. There was a significant difference between M0 and M1a 
$(\mathrm{P}<0.001)$, but no significant difference between $\mathrm{M} 1 \mathrm{a}$ and M1b $(\mathrm{P}=0.092)$ was identified. It has been reported that the oligometastases in this comprehensive treatment mode prolonged the survival time of patients after primary and oligodendrocyte resection. Therefore, our study is in favor of the further division of M1b (thoracic extravasation) in the 7 th edition into M1b and M1c in the 8 th edition for selecting patients eligible for more aggressive topical and systemic treatment.

In conclusion, our study validated the 8th edition UICC staging system in 3,611 surgically treated patients at our institute. Though previous study has evaluated the 8th edition by comparing the OS between the 7 th and 8 th staging systems in general (28), our study explored the survival rate by $T, N$, and $M$ descriptors between the 2 systems. We found that compared to the 7 th edition of the TNM classification, the 8 th edition provides more accurate prognostic information, particularly among lung cancer patients at pathologic stages IA1, IA2, and IA3.

\section{Acknowledgments}

None.

\section{Footnote}

Conflicts of Interest: The authors have no conflicts of interest to declare.

Ethical Statement: The authors are accountable for all aspects of the work in ensuring that questions related to the accuracy or integrity of any part of the work are appropriately investigated and resolved. The study was approved by the Institutional Review Board at the Peking University Cancer Hospital (No. 2017YJZ45). Patient consent was not required since no human subjects participated in our study.

\section{References}

1. de Groot PM, Wu CC, Carter BW, et al. The epidemiology of lung cancer. Transl Lung Cancer Res 2018;7:220-33.

2. Saito S, Espinoza-Mercado F, Liu H, et al. Current status of research and treatment for non-small cell lung cancer in never-smoking females. Cancer Biol Ther 2017;18:359-68.

3. Li S, Ma Y, Xiong Y, Zhang P, et al. Five tumor-associated autoantibodies expression levels in serum predict lung cancer and associate with poor outcome. Transl Cancer Res 2019;8:1364-73.

4. Mirsadraee S, Oswal D, Alizadeh Y, et al. The 7th lung cancer TNM classification and staging system: Review of the changes and implications. World J Radiol 2012;4:128-34.

5. Raptis CA, Bhalla S. The 7th Edition of the TNM staging system for lung cancer: what the radiologist needs to know. Radiol Clin North Am 2012;50:915-33.

6. Palmero R, Navarro-Martin A, Nadal E. Comment on an external validation of the 8th edition of the TNM classification for lung cancer staging in patients treated with chemoradiation. Ann Transl Med 2019;7:S101.

7. Yang L, Wang S, Zhou Y, et al. Evaluation of the 7th and 8th editions of the AJCC/UICC TNM staging systems for lung cancer in a large North American cohort. Oncotarget 2017;8:66784-95.

8. Sui X, Jiang W, Chen H, et al. Validation of the Stage Groupings in the Eighth Edition of the TNM Classification for Lung Cancer. J Thorac Oncol 2017;12:1679-86.

9. Gao Y, Zhang JF, Li QC, et al. The clinicopathological and prognostic features of Chinese and Japanese inpatients with lung cancer. Oncotarget 2016;7:67425-34.

10. Naylor EC, Desani JK, Chung PK. Targeted Therapy and Immunotherapy for Lung Cancer. Surg Oncol Clin N Am 2016;25:601-9.

11. Rami-Porta R, Crowley JJ, Goldstraw P. The revised TNM staging system for lung cancer. Ann Thorac Cardiovasc Surg 2009;15:4-9.

12. Dediu M. Adjuvant chemotherapy in stage IB NSCLC: implication of the new TNM staging system. Memo 2011;4:16-8.

13. Li J, Yang X, Xia T, et al. Stage I synchronous multiple primary non-small cell lung cancer: CT findings and the effect of TNM staging with the 7th and 8th editions on prognosis. J Thorac Dis 2017;9:5335-44.

14. Li DP, Li W, Feng J, et al. Adjuvant chemotherapy with sequential cytokine-induced killer (CIK) cells in stage IB non-small cell lung cancer. Oncol Res 2015;22:67-74.

15. Morgensztern D, Du L, Waqar SN, et al. Adjuvant Chemotherapy for Patients with T2NOM0 NSCLC. J Thorac Oncol 2016;11:1729-35.

16. Graziano SL, Lacas B, Vollmer R, et al. Cross-validation analysis of the prognostic significance of mucin expression in patients with resected non-small cell lung cancer treated with adjuvant chemotherapy: results from IALT, JBR.10 and ANITA. Lung Cancer 2013;82:149-55. 
17. Strauss GM, Herndon JE, Maddaus MA, et al. Adjuvant paclitaxel plus carboplatin compared with observation in stage IB non-small-cell lung cancer: CALGB 9633 with the Cancer and Leukemia Group B, Rad. J Clin Oncol 2008;26:5043-51.

18. Koike T, Kitahara A, Sato S, et al. Lobectomy Versus Segmentectomy in Radiologically Pure Solid SmallSized Non-Small Cell Lung Cancer. Ann Thorac Surg 2016;101:1354-60.

19. Ueki T, Sakaguchi S, Miyajima Y, et al. Usefulness of tumor pressure as a prognostic factor in cases of hepatocellular carcinoma where the diameter of the tumor is $3 \mathrm{~cm}$ or less. Cancer 2002;95:596-604.

20. Ohtaki Y, Shimizu K. Anatomical thoracoscopic segmentectomy for lung cancer. Gen Thorac Cardiovasc Surg 2014;62:586-93.

21. Rami-Porta R, Eberhardt WEE. Clinical implications of the innovations in the primary tumour and metastasis of the 8th edition of the TNM classification for lung cancer. J Thorac Dis 2018;10:S2682-S5.

22. Mueller MR. Tailored management of stage IIIa nonsmall-cell lung cancer in the era of the 8th edition of the TNM classification for lung cancer. Future Oncol2018;14:5-11

23. Christian C, Erica S, Morandi U. The prognostic impact

Cite this article as: Wang J, Wu N, Lv C, Yang Y. External validation of the eighth edition of the TNM classification for lung cancer in 3,611 surgically treated patients at a single institution. Ann Transl Med 2020;8(4):122. doi: 10.21037/ atm.2020.01.128 of tumor size in resected stage I non-small cell lung cancer: Evidence for a two thresholds tumor diameters classification. Lung Cancer 2006;54:185-91.

24. Ahn H, Lee KW, Lee KH, et al. Effect of computed tomography window settings and reconstruction plane on 8th edition T-stage classification in patients with lung adenocarcinoma manifesting as a subsolid nodule. Eur J Radiol 2018;98:130-5.

25. Shin JY, Yoon JK, Marwaha G. External Validation of the New International Association for the Study of Lung Cancer Tumor, Node, and Metastasis 8th Edition Staging System and Updated T Descriptors in Determining Prognosis for Patients With Non-Small Cell Lung Cancer Patients With N3 Disease. Clin Lung Cancer 2017;18:e481-9.

26. Shroff GS, Viswanathan C, Carter BW, et al. Staging Lung Cancer: Metastasis. Radiol Clin North Am 2018;56:411-8.

27. Glastonbury CM, Bhosale PR, Choyke PL, et al. Do Radiologists Have Stage Fright? Tumor Staging and How We Can Add Value to the Care of Patients with Cancer. Radiology 2016;278:11-2.

28. Xizhao S, Wei J, Haiqing C, et al. Validation of the stage groupings in the 8 th edition of the TNM classification for lung cancer. J Thorac Oncol 2017;12:1679-86. 